\title{
Role of aliskiren in cardio-renal protection and use in hypertensives with multiple risk factors
}

\author{
This article was published in the following Dove Press journal: \\ Vascular Health and Risk Management \\ 18 May 2009 \\ Number of times this article has been viewed
}

\author{
Eduardo Pimenta' \\ Suzanne Oparil ${ }^{2}$ \\ 'Endocrine Hypertension \\ Research Center and Clinical \\ Center of Research Excellence \\ in Cardiovascular Disease and \\ Metabolic Disorders, University \\ of Queensland School of Medicine, \\ Princess Alexandra Hospital, \\ Brisbane, QLD, Australia; ${ }^{2}$ Vascular \\ Biology and Hypertension Program, \\ University of Alabama at Birmingham, \\ Birmingham, AL, US
}

\begin{abstract}
The renin-angiotensin-aldosterone system (RAAS) is an important mediator of blood pressure (BP) and volume regulation in both normotensive and hypertensive persons and is a major contributor to hypertension-related target organ damage. The concept of renin inhibition for managing hypertension by blocking the RAAS pathway at its point of activation is very attractive since the renin-angiotensinogen reaction is the first and rate-limiting step in the generation of angiotensin II (Ang II). Aliskiren, the first in a new class of orally effective direct renin inhibitors (DRIs), is approved for the treatment of hypertension. It is effective in reducing BP in the general population of hypertensive patients and in special patient groups such as obese persons, and has a tolerability and safety profile similar to placebo. Aliskiren has renoprotective, cardioprotective and anti-atherosclerotic effects in animal models that appear to be independent of BP lowering. It reduces proteinuria in diabetic patients and has favorable neurohumoral effects in patients with symptomatic heart failure. Additional outcome trials are needed to establish the role of this novel class of antihypertensive medication in the therapeutic armamentarium.
\end{abstract}

Keywords: hypertension, renin inhibitors, renin-angiotensin-aldosterone system

\section{Biology of the renin-angiotensin-aldosterone system}

The renin-angiotensin-aldosterone system (RAAS) is an important mediator of blood pressure (BP) and extracellular fluid volume regulation in both normotensive and hypertensive persons. Renin is an aspartyl protease that is synthesized as a preprohormone, cleaved and stored in an inactive (prorenin) form in the juxtaglomerular cells surrounding the afferent arterioles in the kidney. ${ }^{1}$ Prorenin is rendered enzymatically active by both proteolytic and nonproteolytic processes (Figure 1). Most proteolytic activation of prorenin occurs within the juxtaglomerular cells by cleavage of its 43 amino acid N-terminal pro-segment. ${ }^{1}$ Nonproteolytic activation is a 2-step process that allows prorenin that has been secreted from the juxtaglomerular cells into the circulation to acquire enzymatic activity without removal of the pro-segment. While both prorenin and active renin are secreted from the juxtaglomerular cells into the circulation in response to reductions in glomerular afferent arteriolar pressure, sympathetic nerve stimulation or reduced sodium delivery to the macula densa, prorenin is the predominant circulating form, accounting for approximately $90 \%$ of total renin in normal human plasma and for an even greater portion of the total in diabetic patients. ${ }^{2,3}$

Active renin catalyzes the formation of angiotensin I (Ang I) from angiotensinogen. Ang I, in turn, is processed by angiotensin-converting enzyme (ACE) and other proteases to form angiotensin II (Ang II), an important secretagogue for aldosterone (Figure 2).
Correspondence: Eduardo Pimenta Hypertension Unit, Princess Alexandra Hospital, Ipswich Road, Woolloongabba, Brisbane, QLD, 4I02, Australia

Tel +6I 732405866

Email e.pimenta@uq.edu.au 


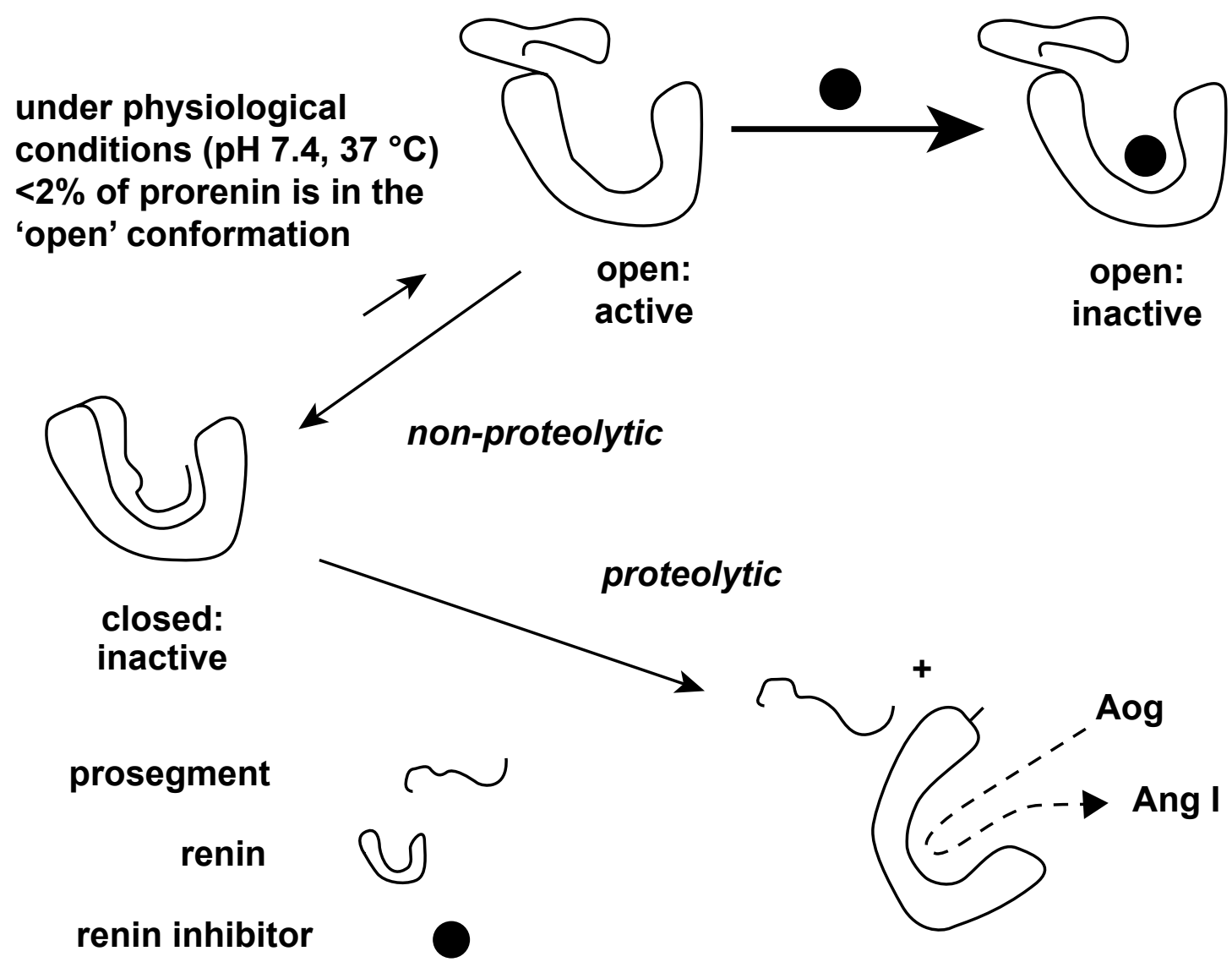

Figure I Proteolytic and non-proteolytic activation of prorenin. A renin inhibitor will increase the amount of nonproteolytically activated prorenin. Such a drug binds to prorenin when it is in its open active conformation. Once bound, the prosegment cannot regain its original "closed" position, and thus prorenin will now be recognized by antibodies directed against the active site, although of course it is incapable of generating angiotensin (Ang) I from angiotensinogen (Aog). Because of the high affinity of the renin inhibitor, prorenin will stay in the "open" conformation, and thus the equilibrium will shift into the direction of the open conformation. Eventually, all prorenin will be in the open conformation. Reproduced with permission From Danser AH, Deinum J. Renin, prorenin and the putative (pro)renin receptor. Hypertension. 2005;46: 1069-1076. ${ }^{7}$ Copyright (c) 2005 Lippincott Williams \& Wilkins.

For the first century of its existence, renin was thought to have no function other than catalysis of the generation of Ang I from angiotensinogen. However, the discovery of a direct receptor for renin and prorenin, referred to as the (pro)renin receptor, has redefined the biology of the RAAS (Figure 2). ${ }^{4}$ Activation of this receptor by renin or prorenin both facilitates angiotensin generation and triggers a signal transduction pathway that is distinct from Ang II receptor signaling. ${ }^{5,6}$ Importantly, binding of prorenin to the (pro)renin receptor changes its configuration and exposes its active site, rendering it fully catalytically active (Figure 1). ${ }^{7}$

Nonproteolytic activation of prorenin can be triggered by binding of its pentameric "handle region" $\left(\mathrm{I}^{11 \mathrm{P}} \mathrm{LLKK}^{15 \mathrm{P}}\right.$ ) to the (pro)renin receptor, resulting in RAAS activation and target organ damage. ${ }^{8,9}$ In the stroke-prone spontaneously hypertensive rat (SHRsp) fed a high salt diet, inhibition of nonproteolytic prorenin activation using a decoy peptide that spans the pentameric handle region has been shown to inactivate the tissue RAAS in heart and to attenuate the development and progression of cardiac fibrosis without affecting the circulating RAAS or arterial pressure. In the kidney of salt fed SHRsp, the majority of nonproteolytically activated prorenin has been localized to the glomerular podocytes, the site at which RAAS activation, proteinuria and glomerulosclerosis occur. In this setting, the decoy peptide completely inhibits nonproteolytic activation of prorenin and activation of the intrarenal RAAS and significantly attenuates the progression of proteinuria and nephrosclerosis without affecting the circulating RAAS or lowering arterial pressure. Taken together, these findings provide strong evidence that (pro)renin receptor mediated nonproteolytic activation of tissue prorenin is an important mediator of both cardiac and renal damage in hypertension.

(Pro)renin receptors have been described in a variety of cell types, including glomerular mesangial cells, human brain, heart, liver, pancreas, kidneys, lung, skeletal muscle placenta, retina, vascular smooth muscle cells and cardiomyocytes. ${ }^{4,6,10}$ Studies using transgenic rats that 


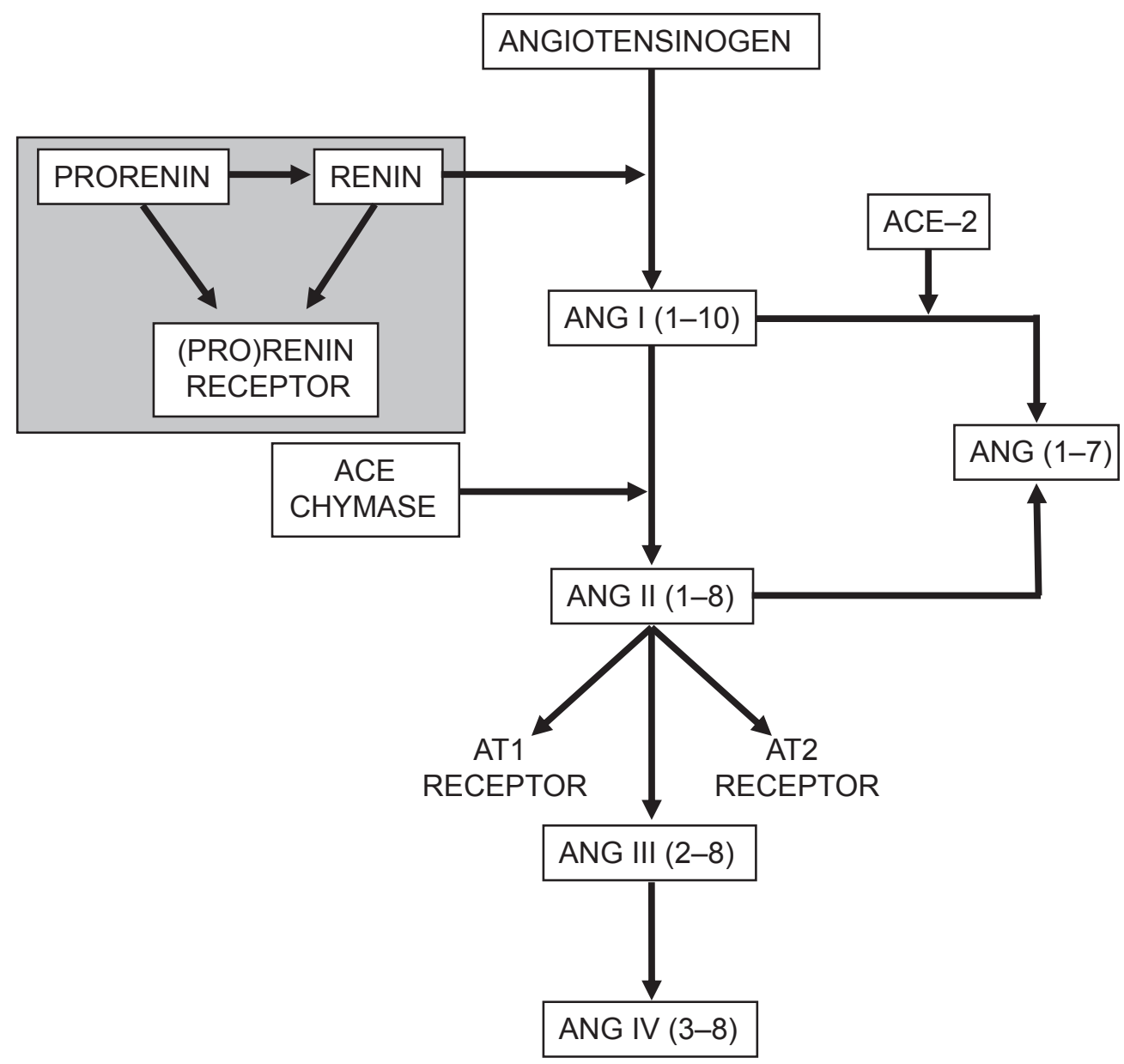

Figure 2 Schematic representation of the renin-angiotensin-aldosterone system. ACE, angiotensin-converting enzyme; Ang, angiotensin (roman numerals refer to the nomenclature for the peptide; numbers in parentheses refer to the amino acid positions in the peptide relative to Ang I, which has I0 amino acids); ATI, angiotensin II type I receptor; AT2, angiotensin II type 2 receptor.Adapted from Reudelhuber TL. Renin. In: Oparil S, Weber MA (eds). Hypertension, 2nd ed.A Companion to Brenner and Rector's The kidney. Philadelphia: Elsevier, 2005, p. 89-94. ${ }^{63}$ Copyright (C) 2005 Elsevier.

express the human (pro)renin receptor genes demonstrate that activation of the (pro)renin receptor can stimulate various intracellular signaling pathways, thus triggering the expression of genes that contribute to BP elevation and related target organ damage. For example, transgenic rats that overexpress the human (pro)renin receptor gene in smooth muscle cells under the control of the mouse smooth muscle myosin heavy chain gene develop a cardiovascular phenotype with elevated systolic BP and heart rate. ${ }^{11}$ These findings are associated with increases in plasma aldosterone and aldosterone/renin ratio in the presence of normal plasma renin, possibly related to the intraadrenal activation of the RAAS.

Binding of human recombinant renin to the (pro)renin receptor in mesangial cells has been shown to provoke phosphorylation of the mitogen-activated protein (MAP) kinases ERK1(p44) and ERK2(p42), which in turn upregulates the expression of profibrotic molecules such as transforming growth factor (TGF)- $\beta$, plasminogen activator inhibitor
(PAI)-1, collagen I and fibronectin., ${ }^{4}$ These effects of (pro)renin receptor activation, which are independent of Ang II, may lead to increased tissue fibrosis and cellular hypertrophy, resulting ultimately in cardiovascular and renal outcomes. ${ }^{13}$ Further, prorenin has been shown to exert angiotensin-independent effects, including stimulation of the p38 MAP kinase/HSP 27 pathway, with resultant alterations in actin filament dynamics, in isolated neonatal cardiomyocytes that express the (pro)renin receptor. ${ }^{10}$ These effects suggest that prorenin, via the (pro)renin receptor, can directly affect cardiac growth and development.

The observation that plasma prorenin levels are associated with the microvascular complications of diabetes, including retinopathy and nephropathy, has suggested that prorenin may contribute to target organ damage in humans. ${ }^{14-16}$ Evidence from streptozotocin diabetic mice has given proof of principle that binding of prorenin to the (pro)renin receptor in kidney can induce diabetic 
nephropathy by an Ang II independent mechanism that does not require proteolytic activation of prorenin. ${ }^{17}$ Building on the observation that the prorenin-to-active renin ratio is increased in diabetes, the investigators tested the hypothesis that prorenin can promote the development of diabetic nephropathy by (pro)renin receptor mediated mechanisms that do not require activation of the RAAS. Wild type and Ang II type 1a receptor gene-deficient (ATKO) mice were treated with streptozotocin to induce diabetes and then with either an ACE inhibitor or the decoy peptide discussed above and the effects of the treatments on progression of diabetic nephropathy were observed. The decoy peptide completely prevented the development of diabetic nephropathy and activation of renal MAP kinases in both wild type and ATKO diabetic mice, demonstrating the AngII independence of its action. In contrast, the ACE inhibitor, administered at doses sufficient to suppress circulating and intrarenal AngII levels, had only a partial effect on the progression of renal disease. Whether these provocative preclinical findings can be translated to the setting of human diabetes is a question for future investigation.

\section{Inhibitors of the renin-angiotensin- aldosterone system}

Increased RAAS activity, particularly increased Ang II and aldosterone levels, contribute to target organ damage and enhance cardiovascular risk both by elevating BP and through direct effects on vascular endothelium and cardiac and renal tissues. ${ }^{3}$ Ang II promotes target organ damage through BP elevation and by mediating constriction and remodeling of resistance vessels, aldosterone synthesis and release, enhancement of sympathetic outflow from the brain, and facilitation of cathecolamine release from the adrenals and peripheral sympathetic nerve terminals. ${ }^{18,19}$ Ang II is also vasculotoxic due to stimulation of oxidative reactions, such as increasing NAD(P)H oxidase, and promotes cardiac and vascular smooth muscle cell hypertrophy by stimulating expression of a number of growth factors and cytokines and their receptors.

Agents that antagonize the RAAS at various steps, including beta blockers, ACE inhibitors, Ang II receptor blockers (ARBs) and aldosterone antagonists, have been used effectively to lower BP, limit or reverse various forms of target organ damage and improve outcomes in patients with hypertension and/or chronic kidney disease, coronary artery disease, left ventricular hypertrophy and heart failure. Direct renin inhibitors (DRIs), developed as the newest class of antihypertensive agents, block the RAAS at its point of origin, the renin-angiotensinogen reaction, and offer a novel approach to the prevention or reversal of target organ damage and cardiovascular events.

\section{Aliskiren}

Aliskiren is the only orally active DRI approved for the treatment of hypertension in humans. ${ }^{20}$ It is a competitive transition state analog and selective inhibitor of human renin. It has a therapeutic potential similar to that of other antagonists of the RAAS. ${ }^{21}$ In humans, the plasma concentration of aliskiren increases dose-dependently after oral administration in doses of 40 to $640 \mathrm{mg} /$ day, peaking after 3 to 6 hours. $^{22}$ The average plasma half-life is 23.7 hours, ranging from 20 to 45 hours, making aliskiren suitable for once-daily administration. The oral bioavailability of aliskiren in humans is limited $(2.7 \%){ }^{22}$ Aliskiren is $47 \%$ to $51 \%$ protein bound and the steady-state plasma concentration is reached after 5 to 8 days of treatment. The main elimination route of aliskiren is via biliary excretion as unmetabolised drug. ${ }^{22}$

Aliskiren is more potent and selective for human renin than the other orally active DRIs, ie, remikiren and enalkiren. It is not metabolized by cytochrome P450, and thus has low potential for significant interactions with other drugs, eg, warfarin, lovastatin, digoxin, valsartan, amlodipine, metformin, celecoxib, atenolol, atorvastatin, ramipril and hydrochlorothiazide (HCTZ). ${ }^{23-26}$

Aliskiren effectively blocks the generation of active renin and of downstream components of the RAAS in both normotensive and hypertensive human subjects. In this respect the DRI differs from the ACE inhibitors and ARBs, which attenuate feedback inhibition of renin synthesis and release by Ang II, resulting in a reactive rise in plasma renin activity (PRA). A double-blind cross-over study in healthy volunteers receiving a low-sodium diet evaluated the effects of 4 oral doses of aliskiren (40, 80, 160, and $640 \mathrm{mg}$ /day) compared with placebo and the ACE inhibitor enalapril $(20 \mathrm{mg})$ on components of the RAAS. ${ }^{22}$ Aliskiren reduced PRA in a dose-dependent manner after both a single oral dose and 8 days of repeated once-daily dosing. The highest doses of aliskiren reduced Ang II levels by a maximum of $89 \%$ and $75 \%$ on days 1 and 8 , respectively, compared with placebo, and aliskiren $\geq 80 \mathrm{mg} /$ day decreased plasma and urinary aldosterone levels by $40 \%$ and $50 \%$, respectively. Enalapril reduced Ang II levels similarly to aliskiren, but increased PRA 15-fold. Later studies confirmed that aliskiren increases plasma renin content, but reduces PRA, presumably by binding to the active site of renin. ${ }^{27}$ Importantly, this study also 
showed that circulating concentrations of prorenin did not increase in response to aliskiren.

The hormonal effects of dual RAAS blockade with aliskiren and the ARB valsartan have been evaluated and compared with the effects of each monotherapy and placebo in both sodium-depleted normotensive individuals and in hypertensive patients ${ }^{28,29}$ Valsartan monotherapy increased PRA, Ang I and Ang II, while PRA, Ang I and Ang II levels with aliskiren monotherapy and with combination therapy were similar to placebo, indicating that the addition of aliskiren to valsartan eliminates the compensatory increase in PRA and Ang II caused by ARBs.

Although aliskiren suppresses PRA, it causes major reactive increases in plasma renin concentration. This has led some to hypothesize that reactive renin secretion may limit the effectiveness of DRIs..$^{30,31}$ They reason that, if the RAAS is at all leaky, allowing even a small percentage of the excess prorenin generated during DRI treatment to be activated, the antihypertensive effect of the DRI may be offset, limiting its utility as an antihypertensive agent. However, this theory is controversial and has been questioned. ${ }^{32}$ Further, Feldman et al recently demonstrated that aliskiren inhibits prorenin by reducing the (pro)renin receptor expression and binding to the active site of prorenin. ${ }^{33}$ It has also been suggested that the DRIs have limited ability to reduce BP in patients with low baseline PRA levels, thus limiting their utility, at least as monotherapy, in the general population of hypertensive patients. ${ }^{31}$ Additional study and clinical experience with aliskiren and other DRIs, as they become available, are needed to validate or refute this hypothesis.

\section{Aliskiren in the treatment of hypertension}

Aliskiren, both as monotherapy and in combination with other agents, has been evaluated extensively in hypertensive patients. ${ }^{29,34,35}$ Aliskiren monotherapy (75-300 mg/day) has a dose-dependent antihypertensive effect that is comparable to that seen with other major classes of antihypertensive drugs and is associated with a placebo level of side effects. ${ }^{36}$ Comparator studies have shown that aliskiren is as effective as HCTZ, the ARBs irbesartan, losartan and valsartan, the calcium channel blocker amlodipine and the beta blocker atenolol and may be slightly more effective than ACE inhibitors in lowering BP. ${ }^{3,37}$ Further reductions in BP are seen when aliskiren is combined with antihypertensive drugs from the other major classes, including RAAS blockers. ${ }^{29}$ Moreover, combination of aliskiren with other antihypertensive agents is a useful strategy in patients who do not respond to aliskiren monotherapy. ${ }^{38}$ For example, in one large study that included almost 900 patients, a single-pill combination of aliskiren with HCTZ provided significantly greater BP reduction than aliskiren alone with similar tolerability. Most recently, aliskiren-based therapy (monotherapy \pm amlodipine if needed) has been shown to be more effective than HCTZ-based therapy in reducing BP in a study of 1124 hypertensive patients who were followed for 1 year. $^{39}$

\section{Aliskiren in special populations Obesity}

There is a strong positive relationship between body mass index (BMI) and the prevalence of hypertension. ${ }^{40,41}$ Risk estimates from the Framingham Heart Study suggest that $65 \%$ to $75 \%$ of cases of hypertension are attributable to overweight and obesity. ${ }^{42}$ Further, hypertension in overweight and obese persons is difficult to control, with reported control rates of less than $20 \% .{ }^{43}$ Pathophysiologic factors in obesity-related hypertension include both volume expansion and activation of the RAAS, suggesting that RAAS blockade might be a beneficial management strategy. ${ }^{44}$ A randomized double-blind study carried out in 489 obese (average BMI $34.4 \mathrm{~kg} / \mathrm{m}^{2}$ ) hypertensive patients that had not achieved BP goals with HCTZ monotherapy compared the antihypertensive efficacy of aliskiren, irbesartan, amlodipine and placebo based treatment added to HCTZ $(25 \mathrm{mg}){ }^{45}$ After 8 weeks of double-blind treatment, aliskiren/HCTZ lowered BP by 15.8/11.9 mm Hg, significantly more than placebo/HCTZ (8.6/7.9 mm Hg). Aliskiren/HCTZ provided BP reductions similar to those with irbesartan/HCTZ and amlodipine/ HCTZ, with similar tolerability to placebo/HCTZ. Adverse event rates were highest with amlodipine/HCTZ because of a higher incidence of peripheral edema. This study demonstrates that combination treatment with aliskiren is an effective and well-tolerated therapeutic option for obese patients with hypertension who fail to achieve BP control with thiazide diuretic treatment, a growing and difficult to manage subgroup of the hypertensive population.

\section{Diabetes}

The RAAS has been implicated in both the pathogenesis and the vascular complications of diabetes. Evidence from the transgenic rat that harbors the mouse renin gene (TG(mRen-2)27), a model of tissue renin overexpression which results in hypertension, insulin resistance, and cardiovascular and renal damage, suggests that local expression of the RAAS in the pancreas 
damages the organ by stimulating NADPH oxidase, thus generating reactive oxygen species. ${ }^{46}$ Treatment of Ren 2 rats with aliskiren has been shown to normalize systemic insulin resistance and islet insulin, Ang II and NADPH activity. If translatable to the human condition, these findings suggest that DRI treatment could prevent diabetes and the components of the metabolic syndrome. As discussed below, preclinical studies have also demonstrated antihypertensive and renoprotective effects of aliskiren in animal models of diabetes.

Hypertension is highly prevalent and relatively resistant to treatment in diabetic patients, necessitating multiple drug treatment in the majority of cases. The antihypertensive efficacy and safety of aliskiren alone and in combination with the ACE inhibitor ramipril have been tested in a double-blind trial carried out in 837 hypertensive diabetic patients. ${ }^{47}$ While all therapies produced significant reductions in 24 hour ambulatory BP, aliskiren monotherapy was superior to ramipril monotherapy in reducing systolic BP and noninferior in reducing diastolic BP. Importantly, the combination provided an additional mean BP reduction of 4.6/2.1 $\mathrm{mmHg}$ over ramipril monotherapy and was well tolerated. These findings suggest that a DRI-ACE inhibitor combination provides an effective and safe therapeutic option for the hypertensive diabetic patient.

\section{Renoprotective effects of aliskiren}

Preclinical studies have shown that aliskiren, like other RAAS inhibitors, has renoprotective effects in both diabetic and nondiabetic models of chronic kidney disease. Aliskiren has been shown to induce a dose dependent decrease in BP and to prevent progressive albuminuria in streptozotocin treated TG(mRen-2)27 rats. ${ }^{33}$ Aliskiren also suppressed renal expression of TGF- $\beta$, collagen I and the (pro)renin receptor in this model. The renoprotective effect of aliskiren in this study was attributed to its extensive partitioning to the kidney, achieving a kidney/plasma concentration ratio of over 60 at 2 weeks of treatment. Extensive binding of aliskiren to glomeruli and small cortical blood vessels was found by autoradiography. Finally, aliskiren was found to bind to the active site of prorenin, suggesting that (pro)renin receptormediated activation of prorenin and the consequent gain in Ang II forming ability may be neutralized by aliskiren. These findings provide evidence that tissue damage related to Ang II generated by (pro)renin receptor-bound, nonproteolytically activated prorenin or prorenin that is proteolytically activated in tissues should be reduced by aliskiren. The authors speculated that aliskiren may inhibit fibrosis in the kidney by suppressing (pro)renin receptor gene expression, thus reducing receptor number and attenuating prorenin-induced profibrotic signaling, by preventing activation of prorenin and by negating the gain in catalytic activity of receptor-bound renin. The role of these mechanisms in the pathogenesis of diabetic nephropathy in either animal models or human subjects remains to be demonstrated.

Renoprotective effects of aliskiren have also been demonstrated in double transgenic rats (dTGR) that express genes for both human renin and angiotensinogen. Aliskiren has been compared to valsartan in preventing target-organ damage in dTGR ${ }^{48}$ Both low- and high-dose aliskiren and high-dose valsartan lowered BP, reduced albuminuria and creatinine levels, attenuated left ventricular (LV) hypertrophy and increased survival in this model. Anti-inflammatory effects of aliskiren and the ARB losartan have also been demonstrated in the kidney of dTGR ${ }^{49}$ In another rat model of advanced diabetic nephropathy, aliskiren reduced albuminuria and other markers of renal damage, including expression of TGF- $\beta$ and collagens III and IV. ${ }^{50}$ When aliskiren was compared with ACE inhibitors or ARBs in these models, the renal and cardiac protective effects were approximately equal. $^{48,50}$

Following the proof of principle elucidated by the animal models, the hypothesis that aliskiren can provide renoprotection by reducing albuminuria in patients with diabetes has been tested. ${ }^{51,52}$ Both 24-hour systolic BP assessed by ambulatory monitoring and urinary albumin/creatinine ratio decreased significantly with aliskiren treatment in a study of 15 patients with type 2 diabetes, hypertension and proteinuria (Figure 3). ${ }^{51}$ The more robust Aliskiren in the Evaluation of Proteinuria in Diabetes (AVOID) study tested whether dual RAAS blockade achieved by adding aliskiren to the maximal recommended dose of an ARB (losartan) and optimal antihypertensive therapy would reduce albuminuria in 599 patients with hypertension, type 2 diabetes and proteinuria at baseline. ${ }^{52}$ Addition of aliskiren to ARB treatment reduced the mean urinary albumin-to-creatinine ratio by $20 \%$, (Figure 4 ), with a reduction of $50 \%$ or more in $24.7 \%$ of the patients. A non-significant $2 / 1 \mathrm{mmHg}$ difference in BP was seen between the treatment groups, suggesting that the renoprotective effect of aliskiren was independent of BP. The total numbers of adverse and serious adverse events were similar in the groups.

It has been suggested that DRIs may provide more complete and thus more effective blockade of the RAAS than standard recommended treatment with ACE inhibitors or ARBs and, therefore, may be more renoprotective. To test this hypothesis, the response of renal plasma flow (RPF), 


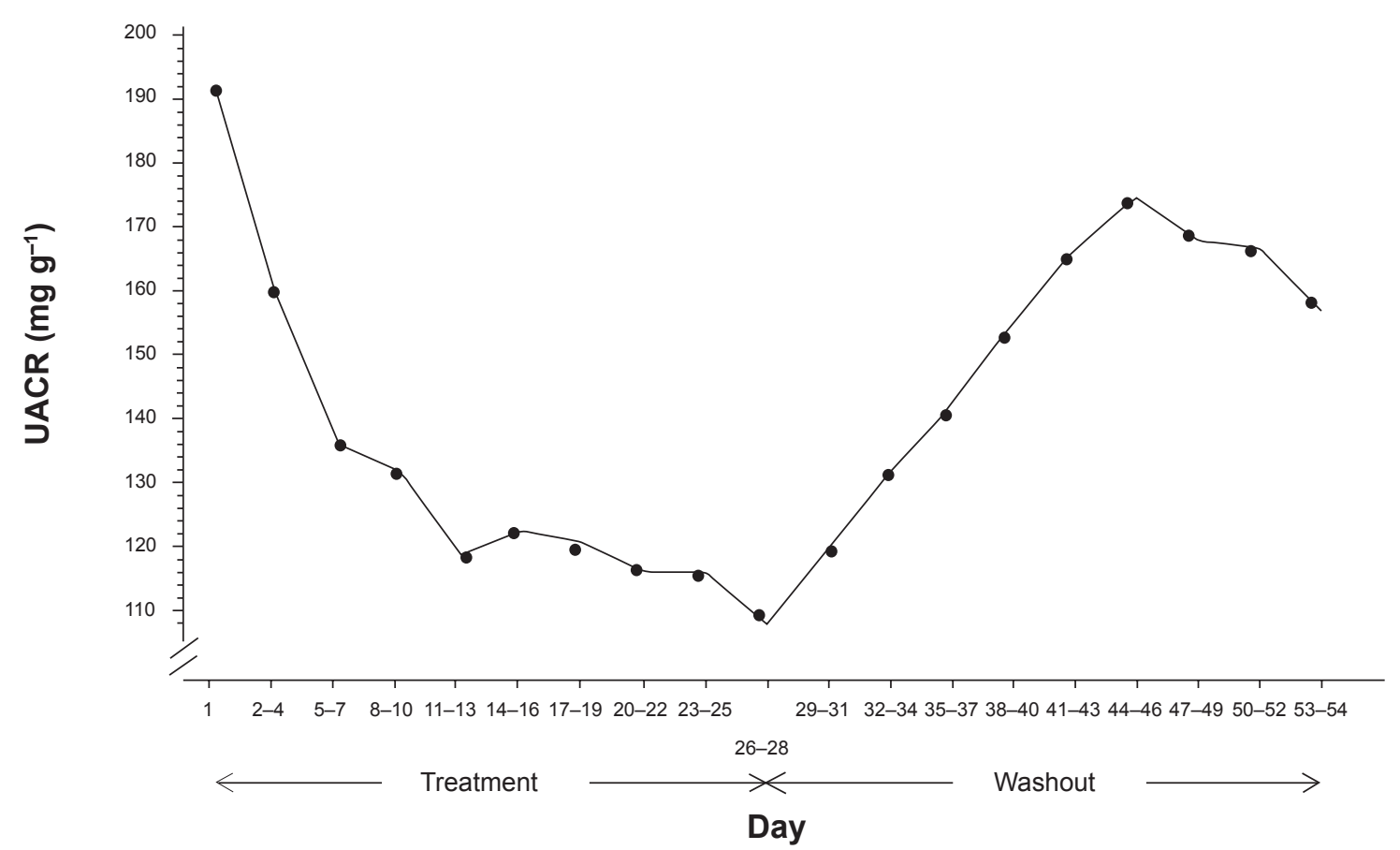

Figure 3 Urinary albumin/creatinine ratio in diabetic patients with micro- or macroalbuminuria treated with aliskiren for 28 days followed by 28 days washout. Reproduced with permission from Persson F, Rossing P, Schjoedt KJ, et al. Time course of the antiproteinuric and antihypertensive effects of direct renin inhibition in type 2 diabetes. Kidney Int. 2008;73: $1419-1425 .{ }^{51}$ Copyright @ 2008 Nature Publishing Group.

a measure of intrarenal renin activity, to treatment with aliskiren or an ACE inhibitor (captopril) was measured in 20 healthy normotensive subjects whose RAAS was activated by consumption of a low-sodium diet. ${ }^{27}$ The RPF response to aliskiren was maximal at the $600 \mathrm{mg}$ dose (twice the maximal recommended dose for hypertension treatment) and exceeded responses to captopril observed in this study, as well as responses seen previously to both ACE inhibitors and ARBs. Residual vasodilation was observed 48 hours after each dose, and aliskiren treatment was associated with significant natriuresis. The authors concluded that DRI treatment promises to provide more complete blockade of the RAAS than treatment with other RAAS blockers and therefore has potential for greater organ protection and improved clinical outcomes, particularly in hypertensive patients with concomitant cardiovascular disease.

\section{Anti-atherosclerotic effects of aliskiren}

Animal experiments and human studies have demonstrated that pharmacological blockade of the RAAS has beneficial effects on atherosclerosis that seem to be independent of BP lowering. ${ }^{53,54}$ The beneficial effects of aliskiren on atherosclerosis progression have been compared to those of a representative ARB (irbesartan), a representative beta blocker (atenolol), and a representative calcium channel blocker (amlodipine) in a mouse model of atherosclerosis. ${ }^{55}$ Two-kidney, 1-clip renovascular hypertension was induced in ApoE-/- mice to generate a model with vulnerable atherosclerotic plaques and 1-kidney, 1-clip renovascular hypertension was induced to generate a model with stable plaques. Aliskiren and irbesartan significantly attenuated atherosclerosis progression in 2-kidney, 1-clip mice compared to untreated animals. Plaques in these animals also showed thinner fibrous caps, smaller lipid cores, decreased media degeneration, layering, and macrophage content, and increased smooth muscle cell content. Aliskiren increased the smooth muscle cell content to a significantly greater extent than irbesartan. If these results are confirmed in clinical studies, patients with clinical or subclinical atherosclerosis could benefit with RAAS blockade with a DRI.

There is also evidence that the DRI aliskiren protects against spontaneously occurring atherosclerosis in the Watanabe heritable hyperlipidemic rabbit by improving endothelial function. ${ }^{56}$ Watanabe rabbits were treated with aliskiren, valsartan, aliskiren plus valsartan or vehicle for 8 weeks and nitric oxide (NO) bioavailability and atherosclerotic plaque area in the aorta were assessed. Acetylcholine-induced NO production, a surrogate index of endothelial protection, was significantly greater with aliskiren+valsartan than with either monotherapy, indicating improvement in endothelial function with 


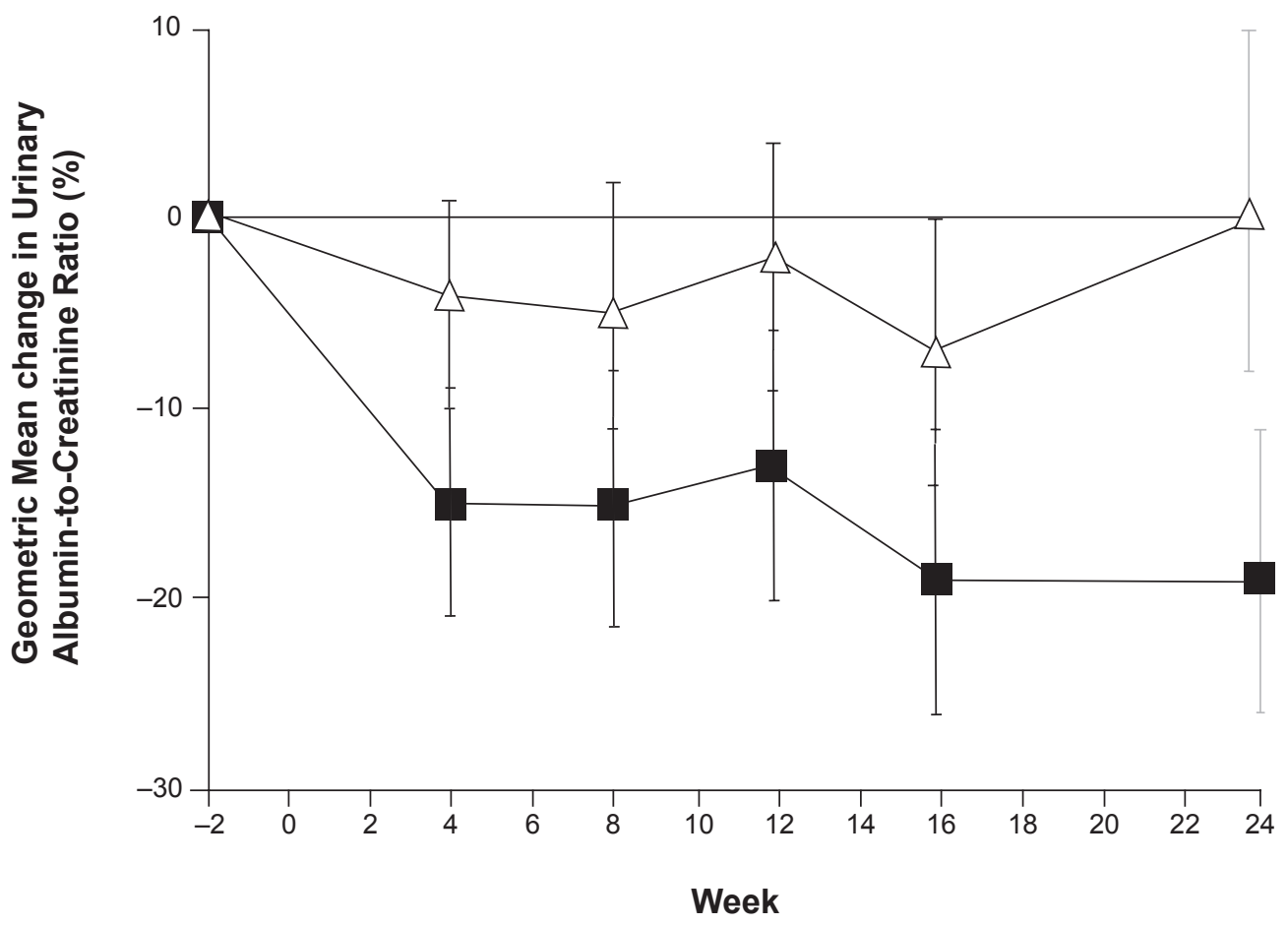

Figure 4 The percentage changes from baseline in the urinary albumin-to-creatinine ratio ( $\mathbf{\square}$ aliskiren; $\Delta$ placebo). Reproduced with permission from Parving $\mathrm{HH}$, Persson $\mathrm{F}$, Lewis JB, et al. Aliskiren combined with losartan in type 2 diabetes and nephropathy. N Engl J Med. 2008;358:2433-2446. ${ }^{52}$ Copyright @ 2008 Massachusetts Medical Society. All rights reserved.

the combination. Similarly, plaque area was decreased to a significantly greater extent with combination therapy compared with either monotherapy.

A recent study in the fat-fed LDL receptor-deficient mouse $\left(L d l r^{-/}\right)$has revealed a novel mechanism by which DRI treatment attenuates the development of atherosclerosis. ${ }^{57}$ Aliskiren treatment produced dose dependent reductions in atherosclerotic lesion size in this model, supporting the RAAS dependence of the atherosclerotic process. To test whether local expression of RAAS components, including renin and the AT1a receptor, in the arterial wall played a role in the progression of atherosclerosis, the authors took advantage of previous observations that macrophages in culture express renin, angiotensinogen and AT1a receptors. To determine whether macrophage expression of renin contributed to the progression of atherosclerosis, they generated chimeric mice with renin deficient bone marrow-derived cells (mostly macrophages) by irradiating $L d l r^{-/}$mice and repopulating with bone marrow-derived cells from renin-- mice. There was a profound decrease in atherosclerotic lesion size in the chimeric mice, supporting a role for local macrophage-derived renin in the pathogenesis of atherosclerosis in this model. In similar experiments, transplantation of bone marrow from ATla receptor ${ }^{-/}$mice failed to influence lesion development, demonstrating that macrophages are not target cells for Ang II in the pathogenesis of atherosclerosis in this model. Subsequent experiments showed that peritoneal macrophages from renin ${ }^{-/}$mice had a significantly reduced ability to induce monocyte adhesion to endothelial cell monolayers, suggesting that endothelial AT1a receptors are targets for macrophage-derived AngII in the setting of murine atherosclerosis. Further study is needed to determine whether these mechanisms are shared by humans.

\section{Cardioprotective effects of aliskiren}

Direct renin inhibition with aliskiren attenuates cardiac dysfunction in animal models. The effect of DRI treatment on cardiac remodeling, apoptosis and LV function after experimental myocardial infarction has been examined in wild type mice that were subjected to coronary artery ligation, then treated with aliskiren or vehicle for 10 days. ${ }^{58}$ Aliskiren treatment improved diastolic and systolic $\mathrm{LV}$ function and decreased post-myocardial infarction hypertrophy, apoptosis, and matrix metalloproteinase activity without altering BP.

There is evidence that aliskiren reduces LV hypertrophy as effectively as an ARB. In the Aliskiren Left Ventricular 
Assessment of Hypertrophy (ALLAY) study, 460 overweight hypertensive patients were randomized to receive aliskiren, losartan, or a combination of the two.${ }^{59}$ Other antihypertensives could be added as necessary. Aliskiren was noninferior to losartan in reducing LV mass index, but the combination was not superior to losartan alone. These findings suggest that aliskiren is an effective and well-tolerated treatment option for patients with LV hypertrophy.

Since loss of negative feedback inhibition of renin release during chronic treatment with ACE inhibitors leads to a compensatory rise in renin secretion and downstream components of the RAAS cascade, thereby possibly attenuating the benefit of the treatment, it has been hypothesized that addition of a DRI to chronic ACE inhibitor treatment would benefit patients with heart failure. The Aliskiren Observation of Heart Failure Treatment (ALOFT) study tested this hypothesis in 302 patients with New York Heart Association class II to IV heart failure and plasma brain-type natriuretic peptide (BNP) concentrations $>100 \mathrm{pg} / \mathrm{mL}$ who were being treated with a beta blocker and an ACE inhibitor or ARB. ${ }^{60}$ Compared with placebo treated controls, patients randomized to aliskiren showed significant improvement in neurohormonal profiles, including reductions in PRA, BNP and N-terminal prohormone BNP (NT-proBNP) levels in plasma and urinary aldosterone concentrations without adverse effects. The findings of ALOFT support further trials to test the safety and efficacy of aliskiren in patients with heart failure, either as an alternative to or in combination with other blockers of the RAAS.

\section{Ongoing studies}

Ongoing studies are testing whether the DRI aliskiren is effective in prevention or regression of various forms of target organ damage in humans. The Safety and Efficacy of Aliskiren in Post Myocardial Infarction Patients (ASPIRE) is evaluating the efficacy and safety of adding aliskiren to optimized standard therapy in the prevention of LV remodeling in post acute myocardial infarction patients. ${ }^{61}$ The Aliskiren Trial in Type 2 Diabetes using Cardiovascular and Renal Disease Endpoints (ALTITUDE) is a placebo controlled trial designed to determine whether adding aliskiren to conventional therapy reduces cardiovascular and renal morbidity and mortality in high risk patients with type 2 diabetes. ${ }^{62}$ ALTITUDE is an event driven trial that aims to randomize 8600 patients with type 2 diabetes who are at high risk because of proteinuria, microalbuminuria accompanied by a reduced estimated glomerular filtration rate (eGFR), or a history of cardiovascular disease accompanied by a reduced eGFR with or without microalbuminuria to aliskiren $300 \mathrm{mg}$ daily or placebo added to usual treatment. Multiple cardiovascular and renal endpoints, including death are planned, and the expected follow-up time will be 48 months.

\section{Conclusion}

Introduction of the DRI aliskiren has opened doors for newer possibilities in the therapy of hypertension and related target organ damage. Aliskiren has antihypertensive efficacy comparable to other classes of antihypertensive medications with a tolerability and safety profile similar to placebo. It also has additive antihypertensive effects when combined with these other drug classes. Preliminary studies of the effects of aliskiren on target organ damage demonstrate comparable or greater efficacy compared to other RAAS antagonists. Results of clinical outcome trials are needed to establish the role of this novel class of antihypertensive medication in the therapeutic armamentarium.

\section{Disclosures}

Dr Pimenta has no conflicts of interest. Dr Oparil has received grants-in-aid from Abbott Laboratories, Astra Zeneca, Boehringer Ingelheim, Bristol Myers-Squibb, Daiichi-Sankyo, Forest Laboratories, GlaxoSmithKline, Novartis, Merck and Co, Pfizer, Sankyo, Sanofi-Aventis, Schering-Plough; has served as consultant for Bristol Myers-Squibb, Daiichi Sankyo, Merck and Co, Novartis, Pfizer, Sanofi Aventis, and The Salt Institute.

\section{References}

1. Reudelhuber TL, Ramla D, Chiu L, et al. Proteolytic processing of human prorenin in renal and non-renal tissues. Kidney Int. 1994;46:1522-1524.

2. Danser AH, Derkx FH, Schalekamp MA, et al. Determinants of interindividual variation of renin and prorenin concentrations: evidence for a sexual dimorphism of (pro)renin levels in humans. J Hypertens. 1998; 16:853-862.

3. Gaddam KK, Oparil S. Renin inhibition: should it supplant ACE inhibitors and ARBS in high risk patients? Curr Opin Nephrol Hypertens. 2008; 17:484-490.

4. Nguyen G, Delarue F, Burckle C, et al. Pivotal role of the renin/prorenin receptor in angiotensin II production and cellular responses to renin. J Clin Invest. 2002;109:1417-1427.

5. Nguyen G. Renin/prorenin receptors. Kidney Int. 2006;69:1503-06.

6. Campbell DJ. Critical review of prorenin and (Pro)renin receptor research. Hypertension. 2008;51:1259-1264.

7. Danser AH, Deinum J. Renin, prorenin and the putative (pro)renin receptor. Hypertension. 2005;46:1069-1076.

8. Ichihara A, Kaneshiro Y, Takemitsu T, et al. Nonproteolytic activation of prorenin contributes to development of cardiac fibrosis in genetic hypertension. Hypertension. 2006a;47:894-900.

9. Ichihara A, Kaneshiro Y, Takemitsu T, et al. Contribution of nonproteolytically activated prorenin in glomeruli to hypertensive renal damage. J Am Soc Nephrol. 2006b;17:2495-2503.

10. Saris JJ, 't Hoen PA, Garrelds IM, et al. Prorenin induces intracellular signaling in cardiomyocytes independently of angiotensin II. Hypertension. 2006;48:564-571. 
11. Burcklé CA, Jan Danser AH, Müller DN, et al. Elevated blood pressure and heart rate in human renin receptor transgenic rats. Hypertension. 2006; 47:552-556

12. Huang Y,Wongamorhtham S, Kasting J, et al. Renin increases messangial cell transforming growth factor-b1 and matrix proteins through receptor-mediated, angiotensin II-independent mechanisms. Kidney Int. 2006;69:105-113.

13. Oliver JA. Receptor-mediated actions of renin and prorenin. Kidney Int. 2006;69:13-15.

14. Danser AH, Van Den Dorpel MA, Deinum J, et al. Renin, prorenin, and immunoreactive renin in vitreous fluid from eyes with and without diabetic retinopathy. J Clin Endocrinol Metab. 1989;68:160-167.

15. Wilson DM, Luetscher JA. Plasma prorenin activity and complications in children with insulin-dependent diabetes mellitus. $N$ Engl J Med. 1990;323:1101-1106.

16. Deinum J, Ronn B, Mathiesen E, et al. Increase in serum prorenin precedes onset of microalbuminuria in patients with insulin-dependent diabetes mellitus. Diabetologia. 1999;42:1006-1010.

17. Ichihara A, Suzuki F, Nakagawa T, et al. Prorenin receptor blockade inhibits development of glomerulosclerosis in diabetic angiotensin II type 1a receptor deficient mice. J Am Soc Nephrol. 2006c;7:1950-1961.

18. Rosendorff $\mathrm{C}$. The renin-angiotensin system and vascular hypertrophy. J Am Coll Cardiol. 1996;28:803-812.

19. Dzau V. Tissue angiotensin and pathobiology of vascular disease: a unifying hypothesis. Hypertension. 2001;37:1047-1052.

20. Gradman AH, Kad R. Renin inhibition in hypertension. J Am Coll Cardiol. 2008;51:519-528.

21. Nussberger J. Renin inhibitors. In Oparil S, Weber MA (eds). Hypertension, A Companion to Brenner and and Rector's The kidney. 2nd ed. Philadelphia. Elsevier. 2005;754-764.

22. Nussberger J, Wuerzner J, Jensen C, et al. Angiotensin II supression in humans by the orally active renin inhibitor aliskiren (SPP100). Comparison with enalapril. Hypertension. 2002;39:e1-e8.

23. Dieterle W, Corynen S, Vaidyanathan S, et al. Effect of the oral renin inhibitor aliskiren on the pharmacokinetics and pharmacodynamics of a single dose of warfarin in healthy subjects. Br J Clin Pharmacol. 2004;58:433-436.

24. Dieterle W, Corynen S, Vaidyanathan S, et al. Pharmacokinetic interactions of the oral renin inhibitor aliskiren with lovastatin, atenolol, celecoxib and cimetidine. Int J Clin Pharmacol Ther. 2005;43:527-535.

25. Dieterich H, Kemp C, Vaidyanathan S, et al. Pharmacokinetic interaction of the oral renin inhibitor aliskiren with hydrochlorothiazide in healthy volunteers. Clin Pharmacol Therap. 2006a;79: P12(abstract).

26. Dieterich H, Kemp C, Vaidyanathan S, et al. Aliskiren, the first in a new class or orally effective renin inhibitors, has no clinically significant drug interactions with digoxin in healthy volunteers. Clin Pharmacol Therap. 2006b;79:P64 (abstract).

27. Fisher ND, Danser J, Nussberger J, et al. Renal and hormonal responses to direct renin inhibition with aliskiren in healthy humans. Circulation. 2008;117:3199-3205.

28. Azizi M, Ménard J, Bissery A, et al. Pharmacologic demonstration of the synergistic effects of a combination of the renin inhibitor aliskiren and the AT1 receptor antagonist valsartan on the angiotensin II-renin feedback interruption. J Am Soc Nephrol. 2004;15:3126-3133.

29. Oparil S, Yarows SA, Patel S, et al. Efficacy and safety of combined use of aliskiren and valsartan in patients with hypertension: a randomized double-blind trial. Lancet. 2007;370:221-229.

30. Sealey JE, Laragh JH. Aliskiren, the first renin inhibitor for treating hypertension: reactive renin secretion may limit its effectiveness. Am J Hypertens. 2007;20:587-597.

31. Sealey JE, Laragh JH. Aliskiren fails to lower blood pressure in patients who have either low PRA levels or whose PRA falls insufficiently or reactively rises. Am J Hypertens. 2009;22:112-121.

32. Ménard J, Azizi M. The difficult conception, birth and delivery of a renin inhibitor: controversies around aliskiren. J Hypertens. 2007;25: 1775-1782.
33. Feldman DL, Jin L, Xuan H, et al. Effects of aliskiren on blood pressure, albuminuria, and (pro)renin receptor expression in diabetic TG(mRen)27 rats. Hypertension. 2008;52:130-136.

34. Villamil A, Chysant SG, Calhoun D, et al. Renin inhibition with aliskiren provides additive antihypertensive efficacy when used in combination with hydrochlorothiazide. J Hypertens. 2007;25:217-226.

35. Andersen K, Weinberger MH, Egan B, et al. Comparative efficacy and safety of aliskiren, an oral direct renin inhibitor, and ramipril in hypertension: a 6-month, randomized, double-blind trial. J Hypertens. 2008;26:589-599.

36. Brown MJ. Aliskiren. Circulation. 2008;118:773-784.

37. Dietz R, Dechend R, Yu CM, et al. Effects of the direct renin inhibitor aliskiren and atenolol alone or in combination in patients with hypertension1. J Renin Angiotensin Aldosterone Syst. 2008;9:163-175.

38. Nickenig G, Simanenkov V, Lembo G, et al. Efficacy of aliskiren/ hydrochlorothiazide single-pill combinations in aliskiren non-responders. Blood Press Suppl. 2008;2:31-40.

39. Schmieder RE, Philipp T, Guerediaga J, et al. Long-term antihypertensive efficacy and safety of the oral direct renin inhibitor aliskiren: a 12-month randomized, double-blind comparator trial with hydrochlorothiazide. Circulation. 2009;119:417-425.

40. Mikhail N, Golub MS, Tuck ML. Obesity and hypertension. Prog Cardiovasc Dis. 1999;42:39-58.

41. Brown CD, Higgins M, Donato KA, et al. Body mass index and the prevalence of hypertension and dyslipidemia. Obes Res. 2000;8:605-619.

42. Garrison RJ, Kannel WB, Stokes J 3rd, Castelli WP. Incidence and precursors of hypertension in young adults: the Framingham Offspring Study. Prev Med. 1987;16:235-251.

43. Bramlage P, Pittrow D, Wittchen HU, et al. Hypertension in overweight and obese primary care patients is highly prevalent and poorly controlled. Am J Hypertens. 2004;17:904-910.

44. Rahmouni K, Correia ML, Haynes WG, et al. Obesity-associated hypertension: new insights into mechanisms. Hypertension. 2005;45:9-14.

45. Jordan J, Engeli S, Boye SW, et al. Direct renin inhibition with aliskiren in obese patients with arterial hypertension. Hypertension. 2007;49:1047-1055.

46. Habibi J, Whaley-Connel A, Hayden MR, et al. Renin inhibition attenuates insulin resistance, oxidative stress, and pancreatic remodeling in the transgenic Ren2 rat. Endocrinology. 2008;149:5643-5653.

47. Uresin Y, Taylor AA, Kilo C, et al. Efficacy and safety of the direct renin inhibitor aliskiren and ramipril alone or in combination in patients with diabetes and hypertension. J Renin-Angiotensin-Aldosterone System. 2007;8:190-200.

48. Pilz B, Shagdarsuren E, Wellner M, et al. Aliskiren, a human renin inhibitor, ameliorates cardiac and renal damage in double-transgenic rats. Hypertension. 2005;46:569-576.

49. Shagdarsuren E, Wellner M, Braesen JH, et al. Complement activation in angiotensin II-induced organ damage. Circ Res. 2005;97:716-724.

50. Kelly DJ, Zhang Y, Moe G, et al. Aliskiren, a novel renin inhibitor, is renoprotective in a model of advanced diabetic nephropaty in rats. Diabetologia. 2007;50:2398-2404.

51. Persson F, Rossing P, SchjoedtKJ, et al. Time course of the antiproteinuric and antihypertensive effects of direct renin inhibition in type 2 diabetes. Kidney Int. 2008;73:1419-1425.

52. Parving HH, Persson F, Lewis JB, et al. Aliskiren combined with losartan in type 2 diabetes and nephropaty. N Engl J Med. 2008;358: 2433-2446.

53. Yusuf S, Sleight P, Pogue J, et al. Effects of an angiotensin-convertingenzyme inhibitor, ramipril, on cardiovascular events in high-risk patients. The Heart Outcomes Prevention Evaluation Study Investigators. N Engl J Med. 2000;342:145-153.

54. Strawn WB, Chappell MC, Dean RH, et al. Inhibition of early atherogenesis by losartan in monkeys with diet-induced hypercholesterolemia. Circulation. 2000;101:1586-1593.

55. Nussberger J, Aubert JF, Bouzourene K, et al. Renin inhibition by aliskiren prevents atherosclerosis progression: comparison with irbesartan, atenolol, and amlodipine. Hypertension. 2008;51:1306-1311. 
56. Imanishi $\mathrm{T}$, Tsujioka $\mathrm{H}$, Ikejima $\mathrm{H}$, et al. Renin inhibitor aliskiren improves impaired nitric oxide bioavailability and protects against atherosclerotic changes. Hypertension. 2008;52:563-572.

57. Lu H, Rateri DL, Feldman DL, et al. Renin inhibition reduces hypercholesterolemia-induced atherosclerosis in mice. J Clin Invest. 2008;118:984-993.

58. Westermann D, Riad A, Lettau O, et al. Renin inhibition improves cardiac function and remodeling after myocardial infarction independent of blood pressure. Hypertension. 2008;52:1068-1075.

59. Solomon S, Appelbaum E, Manning WJ, et al. Effect of the direct renin inhibitor aliskiren, the angiotensin receptor blocker losartan, or both, on left ventricular mass in patients with hypertension and left ventricular hypertrophy. Circulation. 2009;119:530-537.
60. McMurray JJ, Pitt B, Latini R, et al. Effects of the oral direct renin inhibitor aliskiren in patients with symptomatic heart failure. Circ Heart Fail. 2008;1:1-24.

61. Safety and Efficacy of Aliskiren in PostMyocardial Infarction Patients (ASPIRE). Accessed 16 February 2009. URL: http://clinicaltrials.gov/ ct2/show/NCT00414609?term=aliskiren+and+heart+failure \&rank=2.

62. Parving HH, Brenner BM, McMurray JJ, et al. Aliskiren trial in type 2 diabetes using cardio-renal endpoints (ALTITUDE): rationale and study design. Nephrol Dial Transplant. 2009; doi:10.1093/ndt/gfn721.

63. Reudelhuber TL. Renin. In: Oparil S, Weber MA (eds). Hypertension, 2nd ed. A Companion to Brenner and Rector's The kidney. Philadelphia: Elsevier, 2005, p. 89-94.
Vascular Health and Risk Management

\section{Publish your work in this journal}

Vascular Health and Risk Management is an international, peerreviewed journal of therapeutics and risk management, focusing on concise rapid reporting of clinical studies on the processes involved in the maintenance of vascular health; the monitoring, prevention and treatment of vascular disease and its sequelae; and the involvement of

\section{Dovepress}

metabolic disorders, particularly diabetes. This journal is indexed on PubMed Central and MedLine. The manuscript management system is completely online and includes a very quick and fair peer-review system, which is all easy to use. Visit http://www.dovepress.com/ testimonials.php to read real quotes from published authors.

Submit your manuscript here: http://www.dovepress.com/vascular-health-and-risk-management-journal 